\title{
Design and simulation of low voltage RF MEMS series switch array
}

\begin{abstract}
In radio frequency (RF) applications, micro-electromechanical system (MEMS) offers better isolation, low loss, and low power consumption over the electronic switches. The satellite system and telecommunication system require high performance of switching array. In this project, a switching array size of $2 \times 2$ is modeled using 6 units of identical MEMS series switch. The switch array is able to perform connection through 2 input ports and 2 output ports. The simulation is performed using CoventorWare, and the electromagnetic characteristics are simulated using EM3DS. A single series switch has pulled in voltage of $4.5 \mathrm{~V}$, isolation of $-25.3 \mathrm{~dB}$, and insertion loss of $-0.038 \mathrm{~dB}$ at $12 \mathrm{GHz}$. For the overall performances of $2 \times 2$ arrays, the simulation shows insertion loss less than $-0.1 \mathrm{~dB}$, and isolation better than $-40 \mathrm{~dB}$ at $12 \mathrm{GHz}$.
\end{abstract}

Keyword: RF MEMS; Switch array; Isolation; Insertion loss; Voltage actuation 